\title{
Rationale and study design of an early care, therapeutic education, and psychological intervention program for the management of post-intensive care syndrome and chronic pain after COVID-19 infection (PAIN-COVID): study protocol for a randomized controlled trial
}

Antonio Ojeda ${ }^{1}$, Andrea Calvo ${ }^{2 *}$ (D) Tomas Cuñat ${ }^{1}$, Ricard Mellado Artigas $^{2}$, Oscar Comino-Trinidad ${ }^{3}$, Jorge Aliaga ${ }^{3}$, Marilyn Arias $^{3}$, Maribel Ahuir ${ }^{4}$, Carlos Ferrando ${ }^{2,5}$ and Christian Dürsteler ${ }^{1}$

\begin{abstract}
Background: Critically ill patients with COVID-19 are an especially susceptible population to develop post-intensive care syndrome (PICS) due to acute respiratory distress syndrome (ARDS). Patients can suffer acute severe pain and may have long-term mental, cognitive, and functional health deterioration after discharge. However, few controlled trials are evaluating interventions for the prevention and treatment of PICS. The study hypothesis is that a specific care program based on early therapeutic education and psychological intervention improves the quality of life of patients at risk of developing PICS and chronic pain after COVID-19. The primary objective is to determine whether the program is superior to standard-of-care on health-related quality of life at 6 months after hospital discharge. The secondary objectives are to determine whether the intervention is superior to standard-of-care on health-related quality of life, incidence of chronic pain and degree of functional limitation, incidence of anxiety, depression, and post-traumatic stress syndrome at 3 and 6 months after hospital discharge.

Methods: The PAINCOVID trial is a unicentric randomized, controlled, patient-blinded superiority trial with two parallel groups. The primary endpoint is the health-related quality of life at 6 months after hospital discharge, and randomization will be performed with a 1:1 allocation ratio. This paper details the methodology and statistical analysis plan of the trial and was submitted before outcome data were available.

The estimated sample size is 84 patients, 42 for each arm. Assuming a lost to follow-up rate of $20 \%$, a sample size of 102 patients is necessary (51 for each arm).
\end{abstract}

\footnotetext{
*Correspondence: macalvo@clinic.cat

${ }^{2}$ Surgical Intensive Care Unit, Department of Anesthesiology, Critical Care and Pain Treatment, Hospital Clínic, Institut d'investigació August Pi i Sunyer, 08036 Barcelona, Spain

Full list of author information is available at the end of the article
}

C C The Author(s). 2021 Open Access This article is licensed under a Creative Commons Attribution 4.0 International License, which permits use, sharing, adaptation, distribution and reproduction in any medium or format, as long as you give appropriate credit to the original author(s) and the source, provide a link to the Creative Commons licence, and indicate if changes were made. The images or other third party material in this article are included in the article's Creative Commons licence, unless indicated otherwise in a credit line to the material. If material is not included in the article's Creative Commons licence and your intended use is not permitted by statutory regulation or exceeds the permitted use, you will need to obtain permission directly from the copyright holder. To view a copy of this licence, visit http://creativecommons.org/licenses/by/4.0/. The Creative Commons Public Domain Dedication waiver (http://creativecommons.org/publicdomain/zero/1.0/) applies to the data made available in this article, unless otherwise stated in a credit line to the data. 
Discussion: This is the first randomized clinical trial assessing the effectiveness of an early care therapeutic education, and psychological intervention program for the management of PICS and chronic pain after COVID-19. The intervention will serve as proof of the need to implement early care programs at an early stage, having an incalculable impact given the current scenario of the pandemic.

Trial registration: This study is being conducted in accordance with the tenets of the Helsinki Declaration and has been approved by the authors' institutional review board Comité Ético de Investigación Clínica del Hospital Clínic de Barcelona (approval number: HCB/2020/0549) and was registered on May 9, 2020, at clinicaltrials.gov (NCT04394169).

Keywords: COVID-19, Randomized controlled trial, Protocol, Post ICU syndrome, Chronic pain, Critical illness

\section{Introduction}

Since the initial outbreak of COVID-19 in December 2019, there have been more than 14.3 million cases of infection with the SARS-CoV-2 virus reported worldwide [1]. This has led to a large number of hospital admissions testing the capability of many healthcare systems. Among hospitalized patients, $10-20 \%$ are admitted to the intensive care unit (ICU), and more than $70 \%$ of those require invasive mechanical ventilation with an overall mortality over $30 \%$ [2-4].

Critical care survival has been reported as $16-37 \%$, and this will result in an unimaginable size of a cohort of critical care survivors given the number of global infections; these patients can experience a significant worsening of their health status and deterioration of their quality of life [5]. In 2012, the Society of Critical Care defined a new term, the post-intensive care syndrome (PICS), as the worsening of the physical, mental or cognitive patient's status after a critical illness that is maintained beyond hospitalization. Consequently, health-related quality of life (HRQoL) and post-intensive care syndrome (PICS) is becoming the focus of intensive care medicine rather than survival rate alone [6, 7]. As psychological dysfunction can persist for years after ICU discharge, its management is becoming an important strategy to improve quality of life together with early detection of posttraumatic stress disorder and anxiety and depression [8]. Moreover, there is evidence that patients who survive a critical illness have a high prevalence of moderate to extreme chronic pain being an essential factor affecting their ability to return to work and to restore quality of life for up to 5 years following discharge [9]. However, few controlled trials have evaluated interventions for the prevention and treatment of PICS [10].

Patients with COVID-19 are an especially susceptible population to develop PICS due to acute respiratory distress syndrome (ARDS). Patients can suffer acute severe pain and may have long-term deterioration in their mental, cognitive, and functional health after discharge. However, pain is a conscious experience by definition; thus, sedated patients can suffer a high nociceptive input inadvertently [5]. However, there are recent publications describing nervous system involvement after infection with SARS-CoV-2 [11, 12]. Thus, chronic pain could potentially occur as a complication or sequel of COVID19. However, as far as we know, there are no studies related to chronic pain care after a critical illness, specifically in patients with COVID-19 [13].

We hypothesize that a specific care program based on early therapeutic education and a psychological intervention improves the quality of life of patients at risk of developing PICS and chronic pain after COVID-19.

\section{Primary outcome}

The primary objective is to determine if a specific care program based on early therapeutic education and a psychological intervention improves the health-related quality of life (HRQoL) compared to standard care at 6 months after hospital discharge.

\section{Secondary outcomes}

The secondary objectives are to determine if the intervention is superior to standard-of-care, by evaluating:

- The HRQoL at three months after hospital discharge.

- The incidence of chronic pain and the degree of functional limitation at 3 and 6 months after hospital discharge.

- The incidence of anxiety and depression at 3 and 6 months after hospital discharge.

- The incidence of post-traumatic stress syndrome at 3 and 6 months after hospital discharge.

\section{Methods}

\section{Study design}

The PAIN-COVID is a comparative, prospective, singlecenter randomized controlled trial that will include 102 patients (Fig. 1). The trial has been designed in accordance with the fundamental principles established in the Declaration of Helsinki, the Convention of the European Council relating to human rights and biomedicine, and the Universal Declaration of UNESCO on the human genome and human rights, and with the requirements 
Admitted to the ICU due to COVID-19 infection, at least one of the following criteria

1) $\geq 18$ years old,

2) SARS-CoV-2

3) APACHE) II score over 14.

4) ICU stay over 10 days

5) ICU Acquired weakness

5)Delirium during ICU admission.

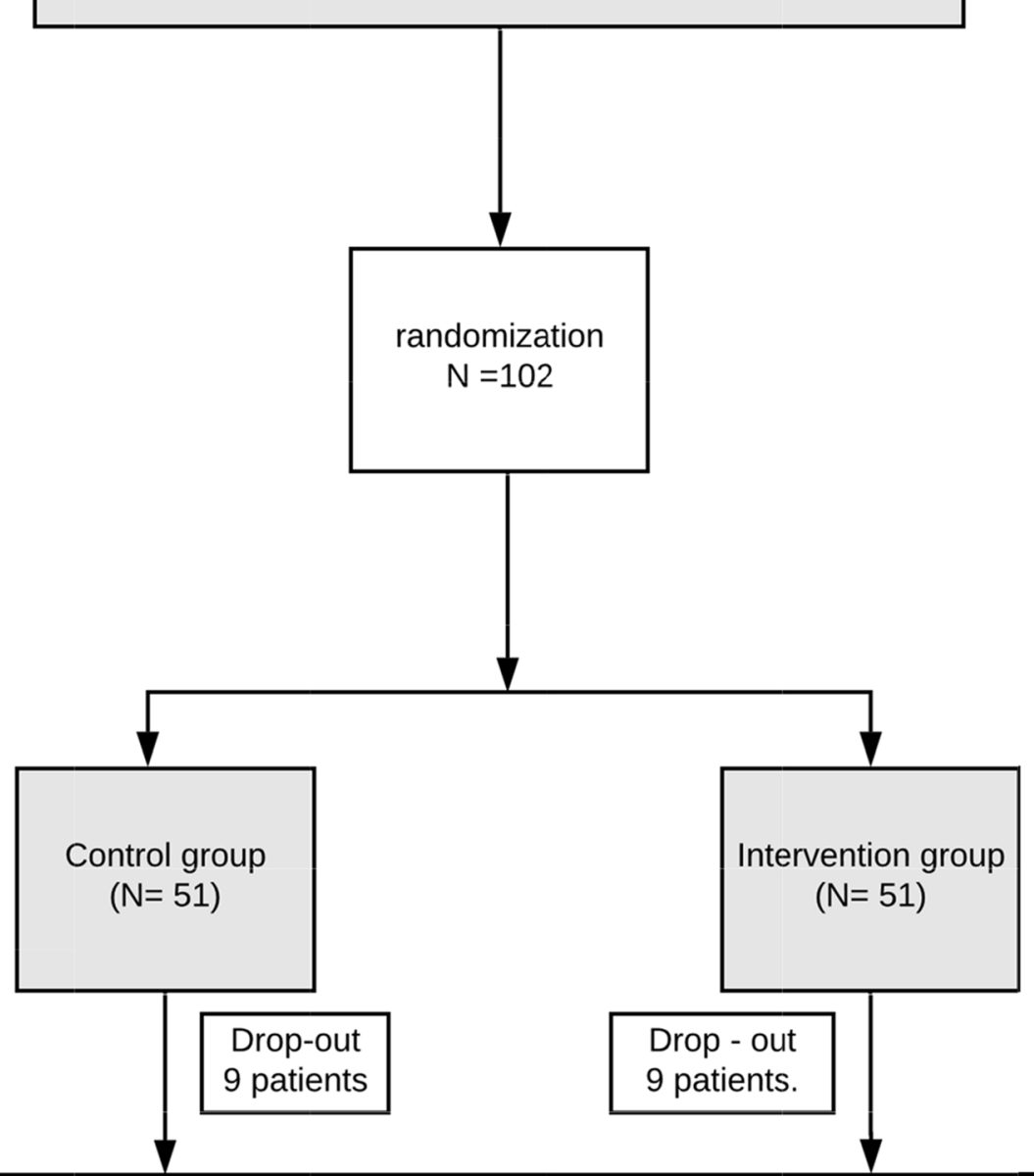

Measurements : Demographic data, Barthel index, MMSE, APACHE II score, SOFA, days of mechanical ventilation, need for tracheostomy, vasoactive drugs, acute renal failure, glycaemia, corticoid use, neuromuscular blocking agents use, sedation days, ICU acquired weakness, delirium during IUC stay, ferritin value, d-dimer and $\mathrm{C}$ reactive protein, ICU and hospital length of stay. Questionnaires EuroQOL-5D-5L, visual analogue scale of the EuroQOL-5D-5L,BPI, DN4,PSC, HAD test ,PCL-5 (baseline visit and at 3 and 6 months after discharge).

Fig. 1 Summary of patient flow diagram

established by Spanish legislation in the field of biomedical research, the protection of personal data, and bioethics, registered on May 9, 2020, at http://www. clinicaltrials.gov with identification No. (NCT04394169). Approval of the final protocol by the Comité Ético de Investigación Clínica del Hospital Clínic de Barcelona- 


\begin{tabular}{|c|c|c|c|c|c|c|c|}
\hline $\begin{array}{r}\text { Time after } \\
\text { Hospital discharqe }\end{array}$ & Enrolment & Allocation & \multicolumn{5}{|r|}{ Close-out } \\
\hline TIMEPOINT** & 1 month & $\begin{array}{c}1-1,5 \\
\text { months }\end{array}$ & $\begin{array}{c}2 \\
\text { mon } \\
\text { ths }\end{array}$ & $\begin{array}{c}3 \\
\text { mon } \\
\text { ths }\end{array}$ & $\begin{array}{l}4 \\
\text { mon } \\
\text { ths }\end{array}$ & $\begin{array}{c}5 \\
\text { mon } \\
\text { ths }\end{array}$ & 6 months \\
\hline ENROLMENT: & Phone call & $\begin{array}{l}\text { Baseline } \\
\text { visit }\end{array}$ & & & & & \\
\hline Eligibility screen & $\mathrm{x}$ & & & & & & \\
\hline Informed consent & $\mathrm{x}$ & & & & & & \\
\hline Allocation & & $\mathrm{x}$ & & & & & \\
\hline INTERVENTIONS: & & & & & & & \\
\hline $\begin{array}{r}\text { Therapeutic } \\
\text { education }\end{array}$ & & $\mathrm{x}$ & $\mathrm{x}$ & & $\mathrm{x}$ & & \\
\hline $\begin{array}{r}\text { Psychological } \\
\text { intervention if met } \\
\text { criteria }\end{array}$ & & $\mathrm{x}$ & & & $\mathrm{x}$ & $\mathrm{x}$ & $\mathrm{x}$ \\
\hline ASSESSMENTS: & & $\mathrm{x}$ & & $\mathrm{X}$ & & & $\mathrm{x}$ \\
\hline baseline variables & & $\mathrm{x}$ & & $\mathrm{x}$ & & & $\mathrm{x}$ \\
\hline $\begin{array}{r}\text { Quality of life } \\
\text { assessment }\end{array}$ & & $\mathrm{x}$ & & $\mathrm{x}$ & & & $\mathrm{x}$ \\
\hline Chronic pain & & $\mathrm{x}$ & & $\mathrm{x}$ & & & $\mathrm{x}$ \\
\hline $\begin{array}{r}\text { Anxiety and } \\
\text { depression } \\
\text { assessment }\end{array}$ & & $\mathrm{x}$ & & $\mathrm{x}$ & & & $\mathrm{x}$ \\
\hline $\begin{array}{r}\text { post-traumatic } \\
\text { disorder } \\
\text { assessment }\end{array}$ & & $\mathrm{x}$ & & $\mathrm{x}$ & & & $x$ \\
\hline
\end{tabular}

Fig. 2 Schedule of enrolment interventions and assessment, SPIRIT 2013

approval number: $\mathrm{HCB} / 2020 / 0549$, Chairperson: Prof Joaquin Fores Viñeta, on May 14, 2020.

This study followed the "Standard Protocol Items: Recommendations for Interventional Trials." The SPIRIT 2013 Statement provides evidence-based recommendations for the minimum content of a clinical trials protocol (Fig. 2).

\section{Study population}

Adult patients will be enrolled if they fulfil at least one of the following Inclusion criteria: (1) had SARS-CoV-2 infection, confirmed with a respiratory tract sample using PCR-based tests, (2) had an Acute Physiology And Chronic Health Evaluation (APACHE II) score over 14, (3) ICU stay over 10 days, (4) acquired weakness in ICU [14] (Supplement, Definition D1), (5) delirium during ICU [14] (Supplement Definition, D2), and 6) acceptance to participate in the study by signing the informed consent form.

The exclusion criteria are as follows: (1) patients with non-confirmed SARS-CoV-2 infection according to WHO guidance [15], (2) central ervous system degenerative diseases or terminal illness (Supplement, Definition D3), (3) terminal illness (Supplement, Definition D4) [16], (4) insufficient understanding of the Spanish language, (5) patients with whom it would be difficult to complete follow-up, and (6) not willing to sign the informed consent form.

\section{Methods of randomization and bias minimization}

Once the informed consent has been obtained, the patient will be assigned 1:1 by the investigator to either the control or intervention group according to the allocation sequence generated by the randomization program. Only the researchers who signed the informed consent had 
access to this list which was concealed from the principal investigator.

The data for the screening will be obtained from the clinical records program of the Hospital Clínic de Barcelona, where the data of patients who required admission to the ICU, as well as days of admission, will be reviewed by the members of the research team in order to verify whether they are eligible or not.

Screening for patients will be done monthly. Enrolment is expected to be completed within 3 to 6 months. The baseline visit will take place between 4 to 6 weeks after hospital discharge. Follow-up visits will take place at 3 and 6 months after discharge (Fig. 2.)

Patients will be encouraged to remain in the study during each interview by giving them feedback about the importance of their collaboration. Participants who decide to drop out during follow-up will be asked about the reason for it, and all their answers will be recorded.

The database will be paper-based and electronic data entry will also be used using FileMaker. Only three research team members will obtain access to this program. case report forms (CRF) will be kept in the hospital files and all data will be available on the web after study finalization.

\section{Blinding}

This is a single-blind study. Visits will be carried out by an investigator with adequate training in questionnaire administration. This investigator will not participate in the intervention or the evaluation of the results. The intervention will be performed by two researchers (a pain physician and a psychologist). These researchers will not participate in the questionnaire and baseline data collection nor in the data analysis. Researchers who analyze the results will not participate in the questionnaire and baseline data collection or program intervention.

\section{General procedures}

The study subjects will be assigned to one of two arms, and the intervention program will be compared to the standard-of-care clinical practice. The baseline visit will take place 4 to 6 weeks after hospital discharge, and two follow-up visits will take place 3 and 6 months after.

The intervention program will consist of an early care therapeutic education on prevention and management of PICS and chronic pain during the three scheduled medical visits within the first 6 months after hospital discharge and psychological treatment for patients at risk of emotional distress.

\section{Recruitment and participant timeline}

Patients who are eligible for the study will be contacted approximately 1 month after discharge from the hospital, they will be informed about the study, and they will be asked to participate. Those who accept to participate will have to complete a baseline visit the following week. Informed consent will be obtained by one of the investigators (Supplement, Figure F2).

\section{Baseline visit}

The baseline visit will take place between 4 to 6 weeks after hospital discharge. Information regarding the study will be given to the patient, and informed consent will be obtained. After that, the patient will be randomized. During this first visit, demographic data, medical history, and ICU and hospitalization variables will be collected from all the included patients, regardless of their randomization arm. All patients will complete a series of questionnaires to evaluate their quality of life and the presence of anxiety, depression, or post-traumatic stress disorder. The presence of pain and its influence on the patients' lifes will also be evaluated.

Intervention group

The intervention consists of a program that includes early patient care, therapeutic education, and psychological intervention. It will be implemented across three medical visits scheduled as follows:

- Visit 1 Intervention group, 4 to 6 weeks after hospital discharge.

- Visit 2 Intervention group, 8 weeks after hospital discharge.

- Visit 3 Intervention group, 18 weeks after hospital discharge.

\section{Components:}

- Interview and physical examination.

- Therapeutic education about the PICS, orally and in writing with specific documents delivered at the end of the visit, i.e., a PICS fact-sheet developed by the investigators and a rehabilitation manual recommended by the Follow-up and Rehabilitation Committee of the Argentine Society of Intensive Care, SATI [14].

- Therapeutic education about pain (if the patient reports pain) which includes an explanation of pain neurophysiology, the rational use of drugs prescribed by other specialists, information about how to manage daily life activities, and the importance of pre-emptive pain management for proper rehabilitation.

A psychological intervention will be conducted if the following criterium is met: a score higher than 8 on the hospital anxiety and depression (HAD) test depression subscale (supplement, questionnaire Q1) [17]. The intervention protocol will consist of 7 weekly sessions lasting 
one and a half hours each (supplement, Table 1). The intervention in depression is based on Rehm's model of self-control. Psychological interventions may cause adverse events resulting in worsening of patients' clinical course (overdose, self-harm, and self-harm attempts). Therefore, the investigators will monitor any related symptom, report it as an adverse event, and refer the patient for treatment by a specialist unit.

\section{Control group}

Standard-of-care is as follows: patient follow-up will be carried out by their referring physicians (primary care physicians or specialists), who will not be involved in the study. After the baseline visit, the second and third visits will be phone call visits at 3 and 6 months after hospital discharge.

\section{Outcome measurements}

Demographic data will be collected at baseline visit, including age, gender, body mass index, smoking habits, socioeconomic level, work status, and marital status. Barthel index and medical history will also be recorded, especially psychiatric disorders, chronic pain, opioid usage, and previous ICU admission (Supplement, Table 2).

Data regarding ICU and hospital admission will be also collected: Acute Physiology and Chronic Health disease Classification System (APACHE II) and Sequential Organ Failure Assessment Score (SOFA) severity scores, days under invasive or non-invasive mechanical ventilation, presence of sepsis [18] (Supplement Definition, D5), need for tracheostomy, use of vasoactive drugs, acute kidney injury (Supplement Definition, D6) and need for renal replacement therapy, stress hyperglycaemia and hypoglycaemia (Supplement Definition, D6, D7), corticoid use, use of neuromuscular blocking agents, days under sedation, ICU acquired weakness, delirium presence, maximum value of ferritin, $d$-dimer and $C$ reactive protein, and ICU and hospital length of stay. The Mini-Mental State Exam (MMSE) test, which is a widely used test of cognitive function among the elderly that assesses orientation, attention, memory, language, and visual-spatial skills, will be evaluated before answering the questionnaires [19].

The impact of the intervention program on healthrelated quality of life reported by the patient will be assessed through the European quality of life 5 dimensions/5 levels (supplement, questionnaire Q2) [20]. The questionnaire assesses the quality of life of study participants according to 5 domains: mobility, self-care, usual activities, pain/discomfort, and anxiety/depression, each scored according to a scale of 1 (no problems) to 5 (extreme problems) and generating a 5-digit code corresponding to the quality of life. The visual analog scale of the same test will also be assessed (from 0-worst imaginable health-to 100-best imaginable health). The questionnaire provides a simple descriptive profile of a respondent's health status. Quality of life will be assessed at Baseline Visit and at 3 and 6 months after discharge.

Pain (presence and intensity) will be assessed by the Brief Pain Inventory (BPI) questionnaire (supplement, questionnaire Q3) [21] at the baseline visit and at 3 and 6 months after discharge. This questionnaire is a multidimensional questionnaire that evaluates pain intensity in the last 24 hours (worst, lowest, average) and current (right now). It also assesses the impact of pain on daily activities (general activity, encouragement, work, social interaction, sleep, enjoyment of life and the ability to walk). The questions are rated on a scale from 0 to 10 , with 10 being the worst possible value. Subsequently, the average intensity score (BPI intensity score) and average interference score (BPI interference score) is calculated. Following IMMPACT recommendations, a clinically significant pain will be recorded if the mean intensity score (BPI intensity score) is greater than or equal to 3 [22].

If BPI is positive for pain, pain catastrophizing will be assessed by the Pain Catastrophizing Scale (PCS) (supplement, questionnaire Q4) [23] and patients will also complete the Douleur Neuropathique en 4 Questions (DN4) (supplement, questionnaire Q5) to screen for neuropathic pain [24]. The PCS consists of 13 questions that explore the frequency of thoughts and feelings that the interviewees have in the presence of current or anticipated pain, which are grouped into three scoring subscales (magnification, rumination and defencelessness). Each question is rated on a 5-point scale (0: not at all; 4: all the time). The maximum total score is 52 points. A score greater or equal than 30 will be considered as a clinically relevant level of catastrophizing.

The impact of the intervention program on the incidence of anxiety or depression will be assessed by the Hospital Anxiety and Depression test (HAD) (supplement, questionnaire Q1) [17], consisting of 14 questions, with two subscales, one for anxiety and the other for depression, with seven items each and a maximum score of 21 for each subscale. The cut-off points are as follows: 0 to 7 imply the absence of clinically relevant anxiety and depression, 8 to 10 imply de presence of symptoms that require consideration, and 11 to 21 it report the presence of relevant symptoms, with a very probable diagnosis of anxiety or depression. According to Bjelland's review, cut-off points equal or greater that 8 will be used as abnormal anxiety or depression's values. This test will be performed at the baseline visit and at 3 and 6 months [25].

Finally, the incidence of post-traumatic stress disorder (PTSD) [26] will be evaluated with the post-traumatic stress disorder checklist questionnaire (PCL-5) [27]. It 
contains 20 questions that correspond to the DSM-V criteria. Participants will rate their symptoms on a scale of 0 (not at all), 1 (slightly), 2 (moderately), 3 (quite) to 4 (extremely), with a score ranging from 0 to 80 . The overall severity of the symptoms can be assessed adding up the scores of each question (interval 0-80). The severity of each symptom can also be evaluated adding up the scores of the questions. DSM- 5 symptom cluster severity scores can be obtained by adding up the scores for the items within a given cluster, i.e., cluster B (items 15), cluster $C$ (items 6-7), cluster D (items 8-14), and cluster E (items 15-20). A provisional PTSD diagnosis can be made by treating each item rated 2 ("moderately") or higher as a symptom endorsed, then following the DSM-5 diagnostic rule which requires at least: $1 \mathrm{~B}$ item (questions 1-5), $1 \mathrm{C}$ item (questions 6-7), $2 \mathrm{D}$ items (questions 8-14), and $2 \mathrm{E}$ items (questions 15-20) (supplement, questionnaire Q6).

For this analysis, questionnaire licensing was obtained. The validated version in Spanish was used for each of them, except for PCL-5 which, being a new questionnaire, is not yet validated in Spanish, but it has the advantage of screening PTSD according to the DSM-V criteria. The questionnaires are shown in the supplement.

\section{Statistical methods \\ Sample size}

To calculate the sample size of the PAIN COVID clinical trial with an assumed average of 50 points on the visual analog scale of the EuroQOL-5D-5L for the control group, and a clinically relevant difference between the groups of $20 \%$, for distribution of a tail with a type I error of 0.05 and a power of $80 \%$, the sample size has been calculated as 84 patients, 42 for each arm. Assuming a loss to follow-up of $20 \%$, the sample size needed is 102 patients (51 in each group).

\section{Data analysis}

Qualitative variables will be presented as proportions, while for quantitative variables, the mean (standard deviation) or median (interquartile range), after checking for normality using the Shapiro-Wilk test, will be used. To compare variables across groups, Student $t$ tests or Mann-Whitney $U$ tests for continuous data and chisquare tests or exact tests for categorical variables will be carried out. Before parametric hypothesis testing, equality of variances will be studied using the Levene's test, and if assumptions are not met, contrasts will be performed with the Welch's test. An intention-to-treat approach will be followed. Two-tailed $P$ values will be presented and a significance level of 0.05 will be used.

For the secondary outcomes, adjustment with the Benjamini-Hochberg procedure will be carried out.
A sub-analysis of the effect of treatment on compliers will be performed for the main outcome. Compliers are defined as those subjects that effectively receive the treatment they are allocated to. For the present study, compliers will be defined as individuals that, having been randomized to the intervention, complete at least two out of three medical visits and at least five out of seven psychological interventions. For the statistical analysis, instrumental variable analysis will be carried out. A twosided probability $(p)$ value of less than 0.05 will be considered to indicate statistical significance. The statistical analysis was performed using R (https://www.rstudio. $\mathrm{com} /$ ) statistical software.

\section{Dissemination policy}

Investigators will communicate the trial results to participants, healthcare professionals, and the general public by posting them in results databases. The purpose of the data collection is specific to achieving the objectives defined in the project. The data collected during the study will be included in the investigators file master owned by the center.

The treatment and communication of personal data of all participants will be in compliance with the Regulation EU 2016/679 of the European Parliament and of the Council of April 27, 2016, on the protection of natural persons as regards the treatment of personal data and the free circulation of data, effective as of May 25, 2018, and to Organic Law 3/2018, of December 5, on the Protection of Personal Data and guarantee of digital rights.

\section{Discussion}

As far as we know, this is the first randomized clinical trial that examines the effectiveness of an early care therapeutic education and psychological intervention for the management of post-intensive care syndrome and chronic pain after COVID-19.

Patients who survive a critical illness often experience disturbances in many areas, with pain being one of the most important topics. Unfortunately, lack of adequate care to manage this pain is often a problem during the patients stay in the units [5]. This study would quantify the impairment on quality life after ICU admission, as well as the incidence of chronic pain, anxiety, depression, and PTSD. The health status derived from our analysis could be used in economic evaluations of healthcare systems and long-term impact and consequences of the pandemic. Likewise, if the effectiveness of our intervention is verified, it would serve as proof of the need to implement early care programs that allow the recovery process to be followed from early stages, given the current scenario of the pandemic.

Being an integral part of this syndrome, and in order to avoid the limitations and effects of pain on the 
patient's quality of life, pain management should be introduced in the ICU.

This project has some limitations.

The main one is that patients meeting inclusion criteria (especially those who have had a more severe course of the disease) may have a severe mobility limitations that would prevent them from attending some trial visits, which would result in a selection bias. This would add to the problem of a small population sample as a consequence of the study being carried out in a single center.

In addition, there are risk factors associated with the development of PICS and chronic pain after a critical illness. These include older age, low socioeconomic status, female gender, previous mental health problems, negative ICU experiences, and delirium.

Therefore, an exploratory analysis of risk factors potentially associated with PICS due to COVID-19 will be carried out, although the sample size will be a determining factor in the conclusions

Future development of multi-center projects may overcome this limitation.

Finally, since this intervention program involves faceto-face visits, the pandemic could limit its correct development.

Restrictions on mobility might make hospital followup visits difficult.

\section{Trial status}

Protocol version number 1.0 (April 29, 2020)

Recruitment status: recruiting ongoing (started: May 27, 2020). Recruitment is anticipated to be completed by September 25, 2020, and study completion by March 25, 2021.

\section{Abbreviations}

PICS: Post-intensive care syndrome; ICU: Intensive care unit; HRQoL: Healthrelated quality of life; ARDS: Acute respiratory distress syndrome; ICUAW: Intensive care unit-acquired weakness; MMSE: Mini-mental state exam; VAS: Visual analog scale; EQOL 5D/5 L: European quality of life 5 dimensions/ 5 levels; BPI: Brief Pain Inventory; HAD: Hospital Anxiety and Depression HAD Scale

\section{Supplementary Information}

The online version contains supplementary material available at https://doi. org/10.1186/s13063-021-05463-7.

\section{Additional file 1}

\section{Acknowledgements}

The authors acknowledge the valuable ongoing collaboration with the questionnaires of EuroQol Research Foundation, eprovide ${ }^{T M}$, Fundació Clínic per a la Recerca Biomèdica IDIBAPS, MD Anderson Symptom Tools. EuroQol Research Foundation did not have any role in the design of the study, data collection, analysis or interpretation of data, nor in the writing of the manuscript.

\section{Authors' contributions}

All authors contributed to the study conception and design. The study design was performed by $A O$. Statistics was designed by RM. AC, AO, and TC drafted the manuscript protocol. Patient recruitment, acquisition, and analysis and interpretation of data will be performed by AC, TC, OC, JA, and MA. CD and CF substantively revised the different contributions of all the authors and collaborated with the structure of this study and submission. All authors read and approved the final version. This is a single-center study, coordinated by Dr. Ojeda and Dr. Calvo (steering committee), responsible for all aspects of the organization, including screening and recruiting of patients. Dr. Ojeda is supervising the trial, and he calls meetings every quarter with the study group ( $A O, A C, R M, T C, O C, J A, M A, C D, C F)$ to evaluate the study, as there is no public involvement group (SPIG) or data monitoring committee overseeing the trial.

\section{Funding}

The authors received no financial support for the research; the payment for the copyright and use of all questionnaire was paid with their own resources

Availability of data and materials

The data that support the findings of this study will be available upon reasonable request to the corresponding author

\section{Declarations}

Ethics approval and consent to participate

The authors certify that this trial was approved by the Ethics Committee of Hospital Clínic de Barcelona_approval number: HCB/2020/0549,

Chairperson: Prof Joaquín Fores Viñeta, on May 14, 2020. The authors will obtain written informed consent to participate and publish the results obtained as described in the protocol before subjects are included in the study.

Consent for publication

Not applicable.

\section{Competing interests}

The authors declare no conflict of interest in relation to this manuscript.

\section{Author details}

${ }^{1}$ Pain Medicine Section, Department of Anaesthesiology, Critical Care and Pain Treatment, Hospital Clínic de Barcelona, Barcelona, Spain. ${ }^{2}$ Surgical Intensive Care Unit, Department of Anesthesiology, Critical Care and Pain Treatment, Hospital Clínic, Institut d'investigació August Pi i Sunyer, 08036 Barcelona, Spain. ${ }^{3}$ Department of Anaesthesiology, Critical Care and Pain Treatment, Hospital Clínic de Barcelona, Barcelona, Spain. ${ }^{4}$ Department of clinical Psychology, Clinical Neuroscience Institute, Hospital Clínic de Barcelona, Barcelona, Spain. ${ }^{5} \mathrm{CIBER}$ de Enfermedades Respiratorias, Instituto de Salud Carlos III, Madrid, Spain

Received: 8 August 2020 Accepted: 16 July 2021

Published online: 24 July 2021

References

1. COVID-19 Map - Johns Hopkins Coronavirus Resource Center [Internet]. [cited 2020 Jul 21]. Available from: https://coronavirus.jhu.edu/map.html

2. Guan W, Ni Z, Hu Y, Liang W, Ou C, He J, et al. Clinical characteristics of coronavirus disease 2019 in China. New Engl J Med. 2020;382(18):1708-20. https://doi.org/10.1056/NEJMoa2002032.

3. Ferrando C, Mellado-Artigas R, Gea A, Arruti E, Aldecoa C, Bordell A, et al. Características, evolución clínica y factores asociados a la mortalidad en UCl de los pacientes críticos infectados por SARS-CoV-2 en España: estudio prospectivo, de cohorte y multi-céntrico. Revista Española de Anestesiología y Reanimación [Internet]. 2020 Jul [cited 2020 Jul 27];S0034935620301870. Available from: https://inkinghub.elsevier.com/retrieve/pii/S0034935620301870

4. Amrein K, Papinutti A, Mathew E, Vila G, Parekh D. Vitamin D and critical illness: what endocrinology can learn from intensive care and vice versa. Endocrine Connections [Internet]. 2018 Dec [cited 2020 May 28];R304-15. Available from: https://ec.bioscientifica.com/view/journals/ec/7/12/EC-18-01 $84 . x \mathrm{ml}$ 
5. Kemp HI, Corner E, Colvin LA. Chronic pain after COVID-19: implications for rehabilitation. NLM (Medline): British journal of anaesthesia; 2020.

6. Elliott D, Davidson JE, Harvey MA, Bemis-Dougherty A, Hopkins RO, Iwashyna TJ, et al. Exploring the scope of post-intensive care syndrome therapy and care. Critical Care Medicine. 2014;42(12):2518-26. https://doi. org/10.1097/CCM.0000000000000525.

7. Inoue S, Hatakeyama J, Kondo Y, Hifumi T, Sakuramoto H, Kawasaki T, et al Post-intensive care syndrome: its pathophysiology, prevention, and future directions. Acute Medicine \& Surgery. 2019;6(3):233-46. https://doi.org/10.1 002 /ams 2.415 .

8. Vlake JH, Van Genderen ME, Schut A, Verkade M, Wils EJ, Gommers D, et al. Patients suffering from psychological impairments following critical illness are in need of information. Journal of Intensive Care. 2020;8(1):6. https://doi. org/10.1186/s40560-019-0422-0.

9. Lee M, Kang J, Jeong YJ. Risk factors for post-intensive care syndrome: a systematic review and meta-analysis. Australian Critical Care. 2020;33:287-94.

10. Kredentser MS, Blouw M, Marten N, Sareen J, Joseph Bienvenu O, Ryu J, et al. Preventing posttraumatic stress in ICU survivors: a single-center pilot randomized controlled trial of ICU diaries and psychoeducation. Critical Care Medicine. 2018;46(12):1914-22. https://doi.org/10.1097/CCM. 0000000000003367.

11. Li H, Xue Q, Xu X. Involvement of the nervous system in SARS-CoV-2 infection. Neurotox Res. 2020:1-7 Available from: https://www.ncbi.nlm.nih. gov/pmc/articles/PMC7220627/. [cited 2020 Jul 28].

12. Ellul MA, Benjamin L, Singh B, Lant S, Michael BD, Easton A, et al. Neurological associations of COVID-19. Lancet Neurology. 2020: S1474442220302210 Available from: https://linkinghub.elsevier.com/retrieve/ pii/S1474442220302210. [cited 2020 Jul 28].

13. Vittori A, Lerman J, Cascella M, Gomez-Morad AD, Marchetti G, Marinangeli F, et al. COVID-19 Pandemic ARDS survivors: pain after the storm? Anesthesia and analgesia. 2020;27:131(1).

14. Busico M, das Neves A, Carini F, Pedace M, Villalba D, Foster C, et al. Followup program after intensive care unit discharge. Medicina Intensiva. 2019; 43(4):243-54. https://doi.org/10.1016/j.medin.2018.12.005

15. Global Surveillance for human infection with coronavirus disease (COVID-19) [Internet]. [cited 2020 Jul 27]. Available from: https://www.who.int/publica tions/i/item/global-surveillance-for-human-infection-with-novel-coronavirus(2019-ncov)

16. SECPAL [Internet]. [cited 2020 Jul 27]. Available from: https://www.secpal. com/biblioteca_guia-cuidados-paliativos-1

17. Herrero MJ, Blanch J, Peri JM, De Pablo J, Pintor L, Bulbena A. A validation study of the hospital anxiety and depression scale (HADS) in a Spanish population. General Hospital Psychiatry. 2003;25(4):277-83. https://doi.org/1 0.1016/S0163-8343(03)00043-4.

18. Singer M, Deutschman CS, Seymour CW, Shankar-Hari M, Annane D, Bauer $M$, et al. The Third International Consensus Definitions for Sepsis and Septic Shock (Sepsis-3). JAMA. 2016;315(8):801-10 Available from: https://www. ncbi.nlm.nih.gov/pmc/articles/PMC4968574/.

19. Creavin ST, Wisniewski S, Noel-Storr AH, Trevelyan CM, Hampton T, Rayment $D$, et al. Mini-Mental State Examination (MMSE) for the detection of dementia in clinically unevaluated people aged 65 and over in community and primary care populations. Vol. 2016. John Wiley and Sons Ltd: Cochrane Database of Systematic Reviews; 2016.

20. Badia X, Roset M, Montserrat S, Herdman M, Segura A. The Spanish version of EuroQol: a description and its applications. European Quality of Life scale. Medicina clinica. 1999;112 Suppl 1:79-85.

21. Brief Pain Inventory (BPI) | MD Anderson Cancer Center [Internet]. [cited 2020 Jul 27]. Available from: https://www.mdanderson.org/research/depa rtments-labs-institutes/departments-divisions/symptom-research/symptom-a ssessment-tools/brief-pain-inventory.html

22. Gewandter JS, Dworkin RH, Turk DC, Farrar JT, Fillingim RB, Gilron I, et al. Research design considerations for chronic pain prevention clinical trials: IMMPACT recommendations. Pain. 2015;156:1184-97.

23. Darnall BD, Sturgeon JA, Cook KF, Taub CJ, Roy A, Burns JW, et al. Development and validation of a daily pain catastrophizing scale. Journal of Pain. 2017;18(9):1139-49. https://doi.org/10.1016/j.jpain.2017.05.003.

24. Perez C, Galvez R, Huelbes S, Insausti J, Bouhassira D, Diaz S, et al. Validity and reliability of the Spanish version of the DN4 (Douleur Neuropathique 4 questions) questionnaire for differential diagnosis of pain syndromes associated to a neuropathic or somatic component. Health and Quality of Life Outcomes. 2007;5(1):66. https://doi.org/10.1186/1477-7525-5-66.
25. Bjelland I, Dahl AA, Haug TT, Neckelmann D. The validity of the Hospital Anxiety and Depression Scale: an updated literature review. Journal of Psychosomatic Research. 2002;52(2):69-77. https://doi.org/10.1016/S0022-3 999(01)00296-3.

26. Ruggiero KJ, Del Ben K, Scotti JR, Rabalais AE. Psychometric properties of the PTSD checklist - civilian version. Journal of Traumatic Stress. 2003;16(5): 495-502. https://doi.org/10.1023/A:1025714729117.

27. Blanchard EB, Jones-Alexander J, Buckley TC, Forneris CA. Psychometric properties of the PTSD checklist (PCL). Behaviour Research and Therapy. 1996;34(8):669-73. https://doi.org/10.1016/0005-7967(96)00033-2.

\section{Publisher's Note}

Springer Nature remains neutral with regard to jurisdictional claims in published maps and institutional affiliations.
Ready to submit your research? Choose BMC and benefit from:

- fast, convenient online submission

- thorough peer review by experienced researchers in your field

- rapid publication on acceptance

- support for research data, including large and complex data types

- gold Open Access which fosters wider collaboration and increased citations

- maximum visibility for your research: over $100 \mathrm{M}$ website views per year

At $\mathrm{BMC}$, research is always in progress.

Learn more biomedcentral.com/submissions 